\title{
PAGED: a pathway and gene-set enrichment database to enable molecular phenotype discoveries
}

\author{
Hui Huang ${ }^{1,2+}$, Xiaogang $\mathrm{Wu}^{1,2,3 \dagger}$, Madhankumar Sonachalam ${ }^{2 \dagger}$, Sammed N Mandape ${ }^{1}$, Ragini Pandey ${ }^{2}$, \\ Karl F MacDorman ${ }^{1}$, Ping Wan ${ }^{4 *}$, Jake Y Chen ${ }^{1,2,3^{*}}$ \\ From Proceedings of the Ninth Annual MCBIOS Conference. Dealing with the Omics Data Deluge \\ Oxford, MS, USA. 17-18 February 2012
}

\begin{abstract}
Background: Over the past decade, pathway and gene-set enrichment analysis has evolved into the study of high-throughput functional genomics. Owing to poorly annotated and incomplete pathway data, researchers have begun to combine pathway and gene-set enrichment analysis as well as network module-based approaches to identify crucial relationships between different molecular mechanisms.

Methods: To meet the new challenge of molecular phenotype discovery, in this work, we have developed an integrated online database, the Pathway And Gene Enrichment Database (PAGED), to enable comprehensive searches for disease-specific pathwways, gene signatures, microRNA targets, and network modules by integrating gene-set-based prior knowledge as molecular patterns from multiple levels: the genome, transcriptome, posttranscriptome, and proteome.

Results: The online database we developed, PAGED http://bio.informatics.iupui.edu/PAGED is by far the most comprehensive public compilation of gene sets. In its current release, PAGED contains a total of 25,242 gene sets, 61,413 genes, 20 organisms, and 1,275,560 records from five major categories. Beyond its size, the advantage of PAGED lies in the explorations of relationships between gene sets as gene-set association networks (GSANs). Using colorectal cancer expression data analysis as a case study, we demonstrate how to query this database resource to discover crucial pathways, gene signatures, and gene network modules specific to colorectal cancer functional genomics.

Conclusions: This integrated online database lays a foundation for developing tools beyond third-generation pathway analysis approaches on for discovering molecular phenotypes, especially for disease-associated pathway/ gene-set enrichment analysis.
\end{abstract}

\section{Background}

Pathway analysis and gene-set enrichment analysis are both widely-used methods to identify significant molecular expression patterns from high-throughput data [1]. Over the last decade, biological pathways have provided natural sources of molecular mechanisms to develop

\footnotetext{
* Correspondence: wanping@mail.cnu.edu.cn; jakechen@iupui.edu † Contributed equally

${ }^{1}$ School of Informatics, Indiana University, Indianapolis, IN 46202, USA

${ }^{4}$ Capital Normal University, Beijing, 100048, China

Full list of author information is available at the end of the article
}

diagnosis, treatment, and prevention strategies for complex diseases [2-4]. The various and massive functional genomics data are effectively analyzed by gene-set enrichment methods instead of individual gene analysis [5-8]. Pathway analysis and molecular signature discovery continue to reveal the association between genotypes and phenotypes, which are simply called molecular profiling or molecular phenotypes. At present, researchers intend to combine pathway and gene-set enrichment approaches and network module-based approaches to

\section{() Biomed Central}

(c) 2012 Huang et al.; licensee BioMed Central Ltd. This is an open access article distributed under the terms of the Creative Commons Attribution License (http://creativecommons.org/licenses/by/2.0), which permits unrestricted use, distribution, and reproduction in any medium, provided the original work is properly cited. 
identify crucial relationships among different molecular mechanisms [1].

As sources of prior knowledge for molecular mechanisms, biological pathway databases are heterogeneous, cross multiple levels, and lack annotations [3]. Different pathway databases may yield divergent results from the same input data. When different databases yield similar results, applying multiple pathway data sources in a single analysis can generate a measure of validation. Unlike candidate pathway analysis, genome-wide pathway analysis does not require prior biological knowledge. In addition, genome-wide pathway analysis can reveal gene interactions across different diseases [3,9] and multiple pathways $[3,10,11]$. Other studies based on an online integrated human pathway database (HPD) also provided associations between different pathways with diverse types, sizes, and sources $[12,13]$ on specific phenotypes. Although these efforts have greatly improved the efficiency of pathway analysis, our knowledge of biological pathways is still far from complete.

Gene signature data from the transcriptome level offers a complementary source of information to complete pathway knowledge. In a recent review, Khatri et al. [1] categorized pathway analysis into three generations of approaches: the first-generation "over-representation analysis" (ORA) approaches, the second-generation "functional class scoring" (FCS) approaches, and the third-generation "pathway topology" (PT) approaches. To overcome the limitations of ORA approaches (gene-level statistics), FCS approaches, such as gene-set enrichment analysis (GSEA) [6], were devised to include overall changes of gene expressions in each pathway/gene set (pathway-level statistics). Third generation approaches also include overall changes of gene expressions based on pathway topology-that is, their upstream/downstream positions within each pathway. Although these third generation approaches were meant to change our understanding of the underlying mechanisms of pathways, they lack information necessary to achieve this: the interdependence between pathways. Annotated knowledge from genome, transcriptome, post-transcriptome, and proteome levels can assist pathway and gene-set enrichment analysis.

Multi-level, multi-scale, knowledge-guided enrichment analysis can enable molecular phenotype discovery for specific human diseases. Currently, the acquisition of prior knowledge and systems modeling poses a challenge for developing tools that go beyond third-generation pathway analysis for disease-specific molecular profiling. Prior knowledge acquisition requires attention to updates and improves the available annotations with descriptive knowledge from multiple levels, especially for information on pathway microenvironment ("condition-, tissue-, and cell-specific functions of each gene") [1,3]. Systems biology modeling must incorporate data from the view of systems biology to build systems with multiple scales, which can be used to generate hypotheses that will give detailed and accurate predictions of changes in systems. Both aspects of this challenge will be addressed by building a database not only containing disease-associated genes, transcript factors, proteins, and microRNAs, but also by organizing their relationships within and between pathways, gene signatures, and any gene sets from existing experiments or papers.

To meet the new challenges of molecular phenotype discovery, we developed in this work an integrated online database, the Pathway And Gene Enrichment Database (PAGED), to enable comprehensive searches for disease-specific pathways, gene signatures, microRNA targets, and network modules, by integrating geneset-based prior knowledge as molecular patterns from multiple levels-the genome, transcriptome, post-transcriptome, and proteome. The new database can provide the following benefits to biological researchers. First, the new database consists of disease-gene association data, curated and integrated from Online Mendelian Inheritance in Man (OMIM) [14] database and the Genetic Association Database (GAD) [15]; therefore, it has the potential to assist human disease studies. Second, as of March 2012 it also contains all current compiled gene signatures in Molecular Signatures Database (MSigDB) [8] and Gene Signatures Database (GeneSigDB) [7]. Third, it further integrates with microRNA-targets from miRecords [16] database, signaling pathways, protein interaction networks, and transcription factor/gene regulatory networks, partially based on data integrated from the Human Pathway Database (HPD) [12] and the Human Annotated and Predicted Protein Interaction (HAPPI) [17] database. All gene sets or pathways are annotated with molecular interaction details whenever available. We integrated the following version of the database OMIM [14] (Feb. 2012), GAD [15] (Aug. 2011), GeneSigDB [7] (v. 4.0, Sept. 2011), MSigDB [8] (v. 3.0. Sept. 2010), HPD [12] (2009), HAPPI [17](v. 1.4) and miRecords [16] (Nov. 2010), which are the latest versions available. An advantage of our work lies in its representation of relationships between pathways, gene signatures, microRNA targets, and/or network modules. These gene-set-based relationships can be visualized as a gene-set association network (GSAN), which provides a "roadmap" for molecular phenotype discovery for specific human diseases. Using colorectal cancer expression data analysis as a case study, we demonstrate how to query PAGED to discover crucial pathways, gene signatures, and gene network modules specific to colorectal cancer functional genomics. 


\section{Methods}

\section{Data sources}

We show an overview of the data integration process in Figure 1. Gene-set data were collected, extracted, and integrated from five major categories. The pathway data sources were from HPD [12], which has integrated 999 human biological pathway data from five curated sources: KEGG, PID, BioCarta, Reactome, and Protein Lounge. The genome-level disease gene relationships were from OMIM [14] and GAD [15]; the transcriptome-level gene signatures were from MSigDB [8] and GeneSigDB [7]; the post-transcriptome-level microRNA data were from miRecords [16]; and the proteome level data was from an integrated protein interaction database
HAPPI [17], which has integrated HPRD, BIND, MINT, STRING, and OPHID databases.

\section{Gene-set data integration}

We treat as gene sets all groups of genes, including disease-associated genes, pathway genes, gene signatures, microRNA-targeted genes, and PPI sub-network modules. The raw files from those data sources have various formats including plaintext, XML, and table. We have written Perl/Java parsers to convert them into a common tab-delimited textual format to ensure syntacticlevel data compatibility. To integrate across different databases, we mapped the gene/protein IDs in all databases to official gene symbols. The gene-set gene data is

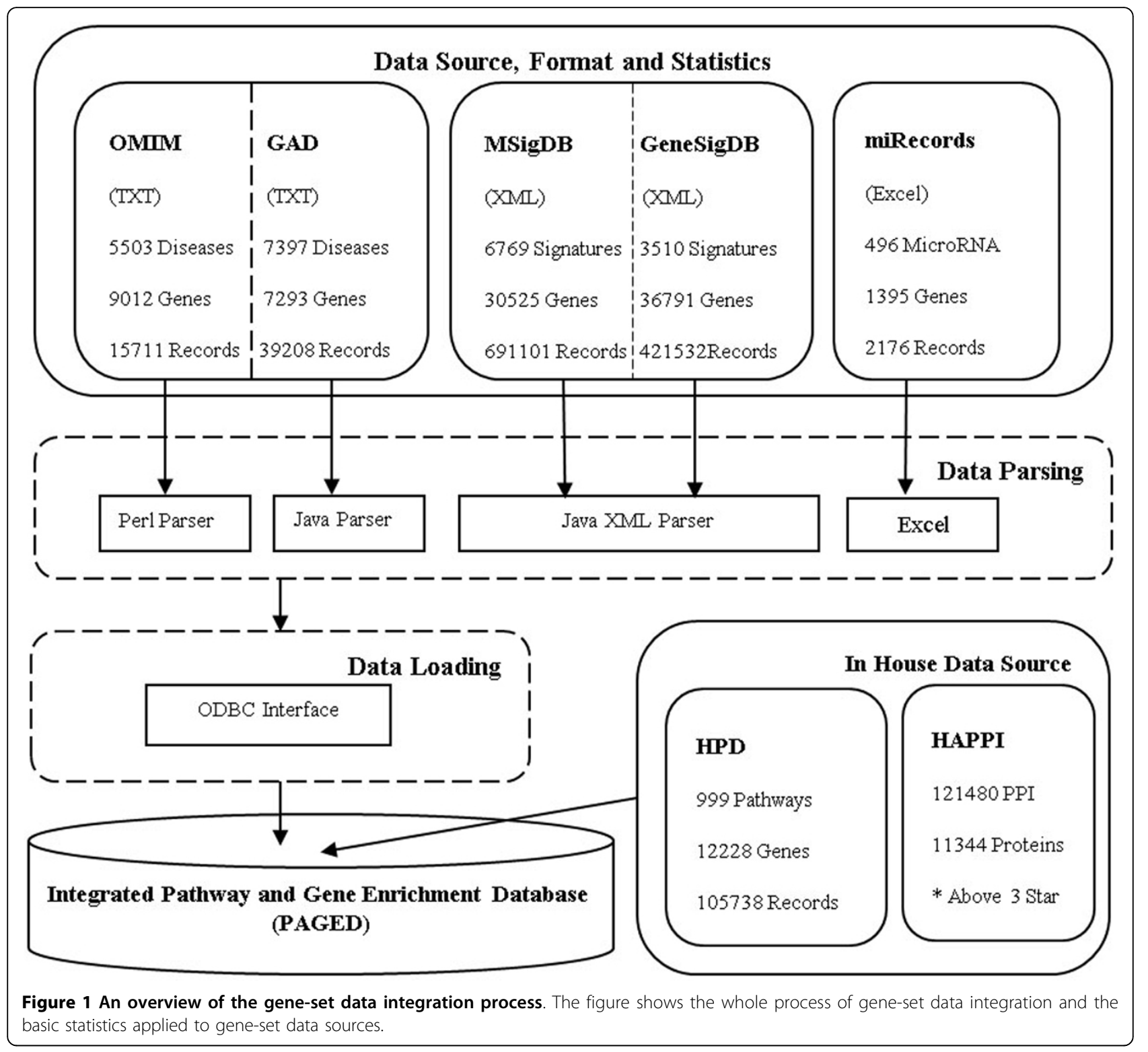


stored in our backend ORACLE11g relational database. As of the current release, PAGED contained a total of 25,242 gene sets, 61,413 genes, 20 organisms, and $1,275,560$ records. All gene set members are represented by the official gene symbols. All PAGED gene sets were assigned unique PAGED-specific identifiers.

\section{Online software designing}

The PAGED platform follows a multi-tiered design architecture. The backend was implemented as PL/SQL packages on an Oracle $11 \mathrm{~g}$ database server and the PAGED application middleware was implemented on the Oracle Application Express (APEX) server, which bridged between the Apache webserver and the Oracle database server.

\section{Gene-set similarity measurement}

Referring to the pathway similarity definition introduced in [12], the similarity score $S_{i, j}$ of two different gene sets is defined by the following formula:

$$
S_{i, j}=\alpha \times S_{L}+(1-\alpha) \times S_{R}=\alpha \times \frac{\left|P_{i} \bigcap P_{j}\right|}{P_{i} \bigcup P_{j}}+(1-\alpha) \times \frac{\left|P_{i} \bigcap P_{j}\right|}{\min \left\{\left|P_{i}\right|,\left|P_{j}\right|\right\}^{\prime}},(i \neq j)
$$

Here, $P_{i}$ and $P_{j}$ denote two different gene sets, while $\left|P_{i}\right|$ and $\left|P_{j}\right|$ are the number of genes in each of these two gene sets. Their intersection $P_{i} \cap P_{j}$ denotes a common set of genes, while their union $P_{i} \cup P_{j}$ is calculated as $\left|P_{i}\right|+\left|P_{j}\right|-\mid$ $P_{i} \cap P_{j} \mid$. Here, $\alpha$ is a weight coefficient among $[0,1]$, which is used to count varying degree of contributions from calculations based both on the overlap (left item $S_{L}$ ) and the cover (right item $S_{R}$ ). $S_{L}$ is well-known as the Jaccard coefficient [18], which is often used to evaluate the similarity between two sets [19]. When a larger gene set covers a smaller one, we expect their similarity score to be high enough to identify them. In this situation, although the left item $S_{L}$ is a small number, the right item $S_{R}$ will be counted as 1.0 to make the final similarity score higher according to our definition in Equation (1), when taking an appropriate $\alpha$ value. Additional file 1 shows that how different $\alpha$ value could affect the distribution of the similarity scores of all cancer related gene sets. We found that when $\alpha$ fell in the interval of $[0.7,0.9]$, the score distribution would be close to a Poisson distribution. As we know, a Poisson distribution expresses the probability of a number of events occurring during a fixed period of time if these events occur with a known average rate and are time-independent since the last event. Therefore, we chose the middle value, $\alpha=0.8$, for the rest of the analysis. Our previous HPD paper [12] also validates the choice of 0.8 as the pathway similarity measurement.

\section{Microarray data}

Here we use colorectal cancer (CRC) expression data analysis as a case study to show how to discover crucial pathways, gene signatures, and gene network modules specific to colorectal cancer functional genomics. We downloaded a colorectal cancer microarray dataset GSE8671 from Gene Expression Omnibus, GEO http:// www.ncbi.nlm.nih.gov/geo/[20]. This microarray dataset compared the transcriptome data of 32 prospectively collected adenomas with those of the normal mucosa from the same individuals. Hence, we have 32 CRC samples and 32 normal samples. We used maximal expression values for the same proteins mapped from different Probe IDs, the Affy package in BioConductor for quantile normalization, the built-in MicroArray Suite (MAS5) for background correction, and Limma in BioConductor for differential analysis, the result of which is represented as fold changes (FC) of CRC samples vs. normal samples.

\section{Differential gene-set expressions}

We use ABS_FC to denote the absolute value of fold change for each gene. We then define differential geneset expressions here as

NORM_ABS_FC: The $p^{*}$-norm of ABS_FC of all the available differential gene expressions in a gene set.

Usually, $p$-norm $=\left(\sum_{\mathrm{i}=1}^{\mathrm{n}}\left(\mathrm{x}_{\mathrm{i}}\right)^{\mathrm{p}}\right)^{\frac{1}{\mathrm{p}}}$

For unification, we modify it as

$$
p^{*} \text {-norm }=\left(\left(\frac{1}{\mathrm{n}} \sum_{\mathrm{i}=1}^{\mathrm{n}}\left(\mathrm{x}_{\mathrm{i}}\right)^{\mathrm{p}}\right)\right)^{\frac{1}{\mathrm{p}}}
$$

In the implementation, $p=6$ performs the best at accentuating highly differential expressions in a gene set.

\section{Gene-set association network (GSAN) construction}

To visualize the relationships between gene sets, we define a gene-set association network (GSAN) as a network of associations between different gene sets, in which the network element representation is as follows:

- Node: Gene set

- Edge: Association between two gene sets

- Node size: Gene-set scale (Counting genes in each gene set)

- Node color: Differential gene-set expression (NORM_ABS_FC)

- Node line color: Gene-set data source

- Edge width: Similarity score

\section{Results}

Database content statistics

Table 1 lists the detailed statistics for each data source and the overlap between each pair. For example, 
Table 1 Number of overlapping genes between different data sources

\begin{tabular}{cccccccc}
\hline & OMIM & GAD & MSigDB & GeneSigDB & miRecords & HPD & HAPP* \\
\hline OMIM & 9012 & 1862 & 3489 & 2792 & 231 & 2559 & 3849 \\
GAD & & 7293 & 6821 & 6450 & 432 & 3202 & 4922 \\
MSigDB & & & 30525 & 17209 & 759 & 6229 & 10677 \\
GeneSigDB & & & & 36791 & 900 & 5904 & 10395 \\
miRecords & & & & & 1395 & 443 & 725 \\
HPD & & & & & & 12228 & 10512 \\
HAPPI & & & & & & & 21955 \\
\hline
\end{tabular}

* Only PPIs of over 3-star quality are considered here; to calculate the overlap, protein IDs from HAPPI have been first converted to gene symbols.

MSigDB contains 30,525 genes and GeneSigDB contains 36,791 genes. The number of overlapping genes between these two databases is 17,209 . We found a synergistic effect from integrating these two signature databases, resulting in greatly increased gene-set coverage. The same effect was observed for all the remaining pair comparisons. These data sources proved to be complementary.

\section{Gene-set scale distributions}

The gene-set scale can reflect the integrality of information content of a biological topic. In this study, we define gene-set scale as the number of molecules (i.e., gene symbols) in a gene set. We performed a statistical analysis of the gene-set scale distributions of both PAGED and of its individual data sources. Figure 2 shows that each data source taken by itself is not very scale-free, especially for OMIM, GAD, and miRecords for higher scales and HPD, GeneSigDB, and MSigDB for lower scales. The scale distribution of PAGED is relatively scale-free on both the low end and the high end with a linear regression $R$-squared of 0.88 . Additionally, the distribution of PAGED always lies above those of its data sources, indicating that the integration has enriched the number of gene sets in all scales instead of exhibiting a bias towards one particular scale. These observations indicate that the integration process of PAGED has resulted in a database that can take account of different gene set scales.

\section{Online functionalities}

In Figure 3, we show the user interfaces of the PAGED website. It supports both disease-based search and userdefined gene-list search. If users search the disease term in the home page (Figure 3A), PAGED will retrieve a list of related gene sets by directly matching the disease term with all the gene-set names; if users instead search a disease term in the advanced search page (Figure 3D), PAGED will first retrieve disease-relevant genes from OMIM and GAD and then use those genes to query the whole database, which will retrieve a gene-set list based

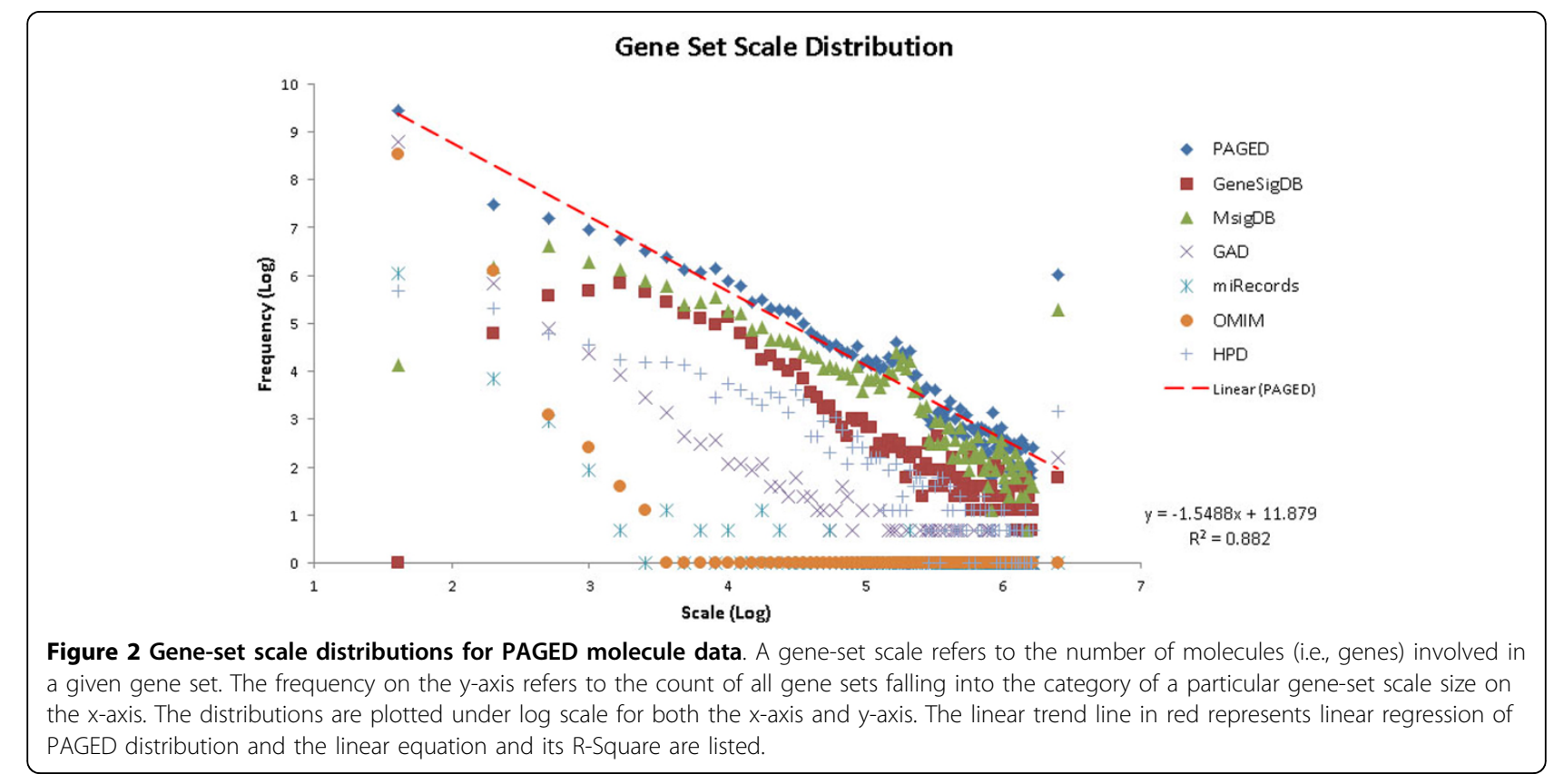




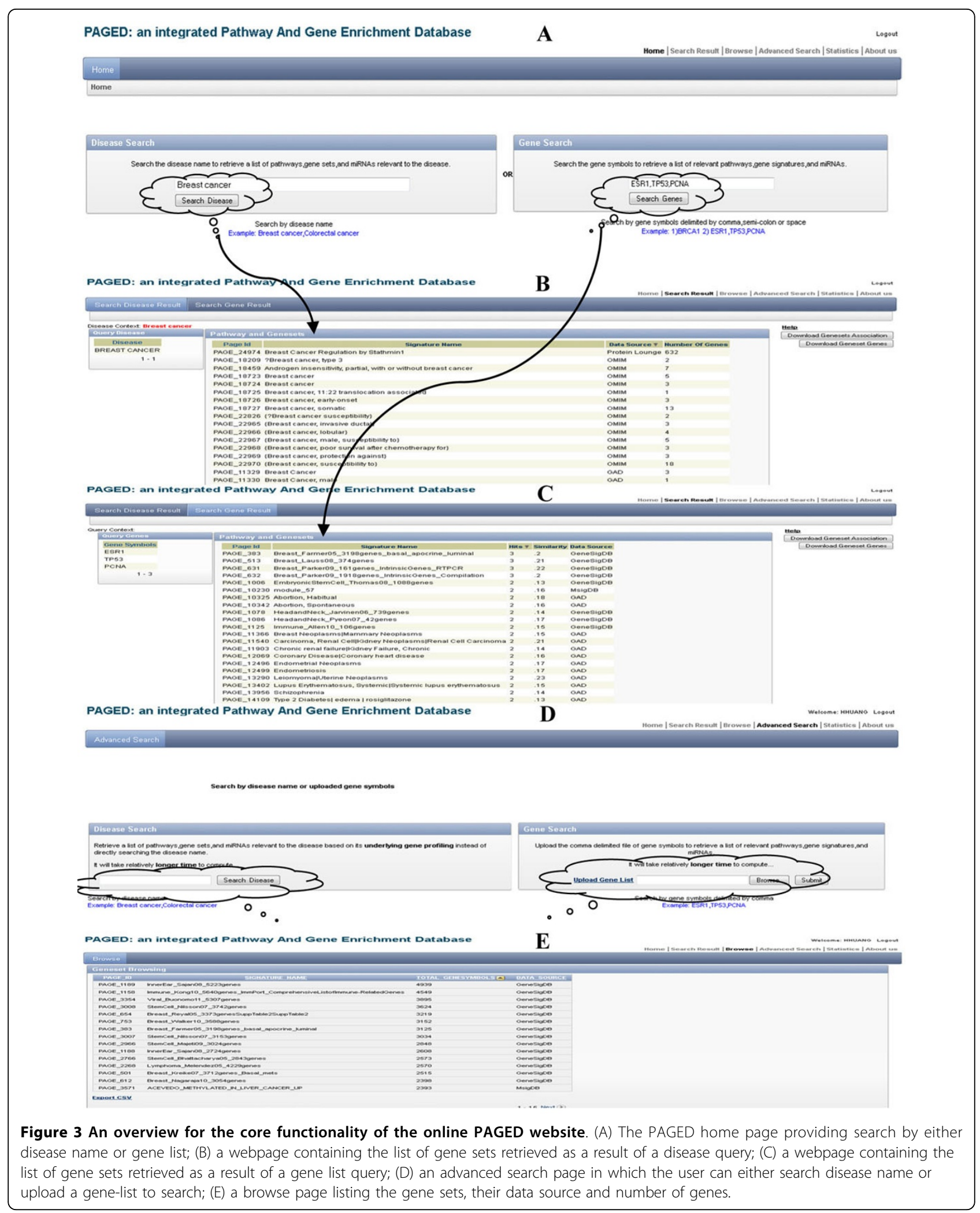


on disease gene profiles that is more comprehensive than that of either OMIM or GAD individually. Users can also search PAGED using multiple genes in the home page (by delimiting them with a comma) to retrieve a list of related gene sets with the hits number and similarity scores (Figure 3A). In addition, users can upload a file of their genes with one gene per line on the advanced search page (Figure 3D) to perform the gene-based search. In the advanced gene-based search (Figure 3D), user can also perform an organism specific search though the majority of the gene-sets are human related. All the gene sets are hyperlinked to the original database, where user can further examine the detailed annotations of that specific gene set.

Upon executing the queries, PAGED can retrieve a list of related gene sets in an HTML table (Figure 3B, C) with their specific organism information included, which are downloadable as a comma-separated value (CSV) file. On the same page, there are links for downloading all the genes in those gene sets and the association between each gene set. In the gene set association downloading page, a simple heat map is provided for the visualization of gene set similarities. More sophisticated visualization will be provided in the near future.

\section{Case studies}

The following case studies use colorectal cancer expression data analysis as a case study to demonstrate how to discover crucial pathways, gene signatures, and gene network modules specific to colorectal cancer functional genomics.

\section{Case study I: Searching disease-associated gene sets based} on gene-set names

Using the standard query box provided at the PAGED home page, one can search for colorectal cancer in all biological gene sets. PAGED returns a list of gene sets, which can be ordered by decreasing number of genes contained by each gene set. In total, 45 gene sets from three data sources (i.e., OMIM, GAD and KEGG) have been retrieved. Not surprisingly, most of them are disease-related gene sets from either OMIM or GAD. Only 1 (i.e., "Colorectal cancer pathway") out 45 is from KEGG. The top 10 search results are listed in Table 2.

Case study II: Searching disease-associated gene sets based on gene-set components

Next, a user can search with the same term colorectal cancer on the advanced search page, which uses the disease's gene profile to search for gene sets. PAGED first obtained 203 colorectal cancer related genes from OMIM and GAD. Then, it used those genes to retrieve a total of 4,932 gene sets with at least 2 hits. Since we are more interested in gene sets other than disease terms, we excluded those gene sets from OMIM and GAD for further analysis. To rule out the possibility
Table 2 Top 10 search results by querying colorectal cancer at the home page

\begin{tabular}{lll}
\hline Gene-set Name & $\begin{array}{l}\text { \# of } \\
\text { Genes }\end{array}$ & $\begin{array}{l}\text { Data } \\
\text { Source }\end{array}$ \\
\hline colorectal cancer & 433 & GAD \\
Colorectal cancer & 134 & KEGG \\
Colorectal cancer & 14 & OMIM \\
Colorectal cancer, somatic & 12 & OMIM \\
Colorectal cancer, hereditary non-polyposis, & 7 & OMIM \\
type 8 & & \\
Colorectal cancer, susceptibility to & 7 & OMIM \\
$\begin{array}{l}\text { Colorectal cancer, hereditary non-polyposis, } \\
\text { type 6 }\end{array}$ & 6 & OMIM \\
Breast and colorectal cancer, susceptibility to & 5 & OMIM \\
Colorectal Cancer & 5 & GAD \\
\hline
\end{tabular}

that those gene sets were hit randomly, we did a Fisher's exact test to calculate the $p$-value between those 203 genes and every retrieved gene set. Finally, we obtained 3,879 gene sets with a $p$-value $<0.05$ and hits $\geq 2$. These gene sets are from all data sources, including MSigDB, GeneSigDB, miRecords, and all pathway data sources from HPD. Both the number of gene sets and their variety support the conclusion that advanced disease search based on gene profiles are more comprehensive than a simple disease search.

Table 3 shows the top results ranked by decreasing number of hits from each data source. Protein Lounge suggests "Molecular Mechanisms of Cancer," "Akt Signaling," and other important pathways in colorectal cancer; BioCarta suggests "wnt signaling pathway"; and NCI Nature curated suggests "Canonical Wnt signaling pathway." These are all very important pathways in colorectal cancer development [21]. Similarly, "Colorectal cancer" and "p53 signaling pathway" from KEGG, "SIGNAL_TRANSDUCTION" and "KEGG_PATHWAYS_IN_CANCER" from MSigDB, and cancer-related signatures/microRNA from GeneSigDB/miRecords from Table 3 reveal a comprehensive picture of the important gene sets involved in colorectal cancer. Thus, the results of the advanced search yield more insights about colorectal cancer mechanisms than those of the simple search.

\section{Case study III: Searching gene sets similar to user-defined query gene sets}

To use the gene-based search from PAGED, we first analyzed a colorectal cancer microarray dataset GSE8671 with BioConductor to identify the differential genes. We selected the top 100 genes ranked by the absolute fold change with $p$-values less than 0.05 . After querying PAGED with those 100 genes, we obtained 1,707 gene sets, out of which 1,152 also satisfied Fisher's exact test of a $p$-value less than 0.05 . Those gene sets span from all the data sources except BioCarta and miRecords. Table 4 lists the top results ranked by the number of hits. Most of 
Table 3 Top search results of colorectal cancer advanced search

\begin{tabular}{|c|c|c|c|c|}
\hline Gene-set Name & Hits & $P$ value & FDR & Data Source \\
\hline Molecular Mechanisms of Cancer & 38 & $2.48 \mathrm{E}-17$ & $7.04 \mathrm{E}-10$ & Protein Lounge \\
\hline PI3K Signaling & 33 & $2.01 \mathrm{E}-13$ & $7.04 \mathrm{E}-10$ & Protein Lounge \\
\hline Akt Signaling & 27 & $9.6 \mathrm{E}-13$ & 7.04E-10 & Protein Lounge \\
\hline ERK Signaling & 24 & $1.53 \mathrm{E}-10$ & $7.04 \mathrm{E}-10$ & Protein Lounge \\
\hline GSK3 Signaling & 23 & $1.32 \mathrm{E}-13$ & $7.04 \mathrm{E}-10$ & Protein Lounge \\
\hline inactivation of gsk3 by akt causes accumulation of b-catenin in alveolar macrophages & 9 & 3.7E-11 & $7.04 \mathrm{E}-10$ & BioCarta \\
\hline atm signaling pathway & 8 & $6.28 \mathrm{E}-11$ & $7.04 \mathrm{E}-10$ & BioCarta \\
\hline wnt signaling pathway & 7 & 7.7E-09 & $7.04 \mathrm{E}-10$ & BioCarta \\
\hline cell cycle: g2/m checkpoint & 7 & 2.14E-08 & 7.04E-10 & BioCarta \\
\hline cell cycle: $g 1 / s$ check point & 7 & $2.14 \mathrm{E}-08$ & $7.04 \mathrm{E}-10$ & BioCarta \\
\hline Canonical Wnt signaling pathway & 8 & $9.24 \mathrm{E}-10$ & $7.04 \mathrm{E}-10$ & NCl-Nature \\
\hline Presenilin action in Notch and Wnt signaling & 8 & $3.16 \mathrm{E}-08$ & $7.04 \mathrm{E}-10$ & NCl-Nature \\
\hline Plasma membrane estrogen receptor signaling & 7 & $1.41 \mathrm{E}-08$ & $7.04 \mathrm{E}-10$ & NCl-Nature \\
\hline FOXM1 transcription factor network & 7 & $2.48 \mathrm{E}-07$ & 7.04E-10 & NCl-Nature \\
\hline LPA receptor mediated events & 7 & $1.45 \mathrm{E}-06$ & $7.04 \mathrm{E}-10$ & NCl-Nature \\
\hline Metabolism of xenobiotics by cytochrome P450 & 20 & $3.3 \mathrm{E}-25$ & $7.04 \mathrm{E}-10$ & KEGG \\
\hline Drug metabolism - cytochrome P450 & 17 & 4.96E-21 & $7.04 \mathrm{E}-10$ & KEGG \\
\hline Bladder cancer & 15 & $3.29 \mathrm{E}-18$ & $7.04 \mathrm{E}-10$ & KEGG \\
\hline Cytokine-cytokine receptor interaction & 15 & 1.39E-06 & $7.04 \mathrm{E}-10$ & KEGG \\
\hline Colorectal cancer & 14 & 4.43E-14 & $7.04 \mathrm{E}-10$ & KEGG \\
\hline p53 signaling pathway & 14 & 4.92E-14 & $7.04 \mathrm{E}-10$ & KEGG \\
\hline Prostate cancer & 14 & $1.66 \mathrm{E}-12$ & $7.04 \mathrm{E}-10$ & KEGG \\
\hline Xenobiotics & 5 & 3.32E-08 & 7.04E-10 & Reactome \\
\hline Formation of incision complex in GG-NER & 5 & $5.75 \mathrm{E}-06$ & $7.04 \mathrm{E}-10$ & Reactome \\
\hline Global Genomic NER (GG-NER) & 5 & 5.75E-06 & 7.04E-10 & Reactome \\
\hline Dual incision reaction in GG-NER & 5 & $5.75 \mathrm{E}-06$ & $7.04 \mathrm{E}-10$ & Reactome \\
\hline Exocytosis of Alpha granule & 5 & 0.000217 & $1.95 \mathrm{E}-08$ & Reactome \\
\hline SIGNAL_TRANSDUCTION & 55 & $8.36 \mathrm{E}-28$ & $7.04 \mathrm{E}-10$ & MsigDB \\
\hline BIOPOLYMER_METABOLIC_PROCESS & 49 & 4.16E-22 & $7.04 \mathrm{E}-10$ & MsigDB \\
\hline KEGG_PATHWAYS_IN_CANCER & 43 & $9.9 \mathrm{E}-46$ & $7.04 \mathrm{E}-10$ & MsigDB \\
\hline NUCLEOBASENUCLEOSIDENUCLEOTIDE_AND_NUCLEIC_ACID_METABOLIC_PROCESS & 41 & $2.16 \mathrm{E}-20$ & 7.04E-10 & MsigDB \\
\hline NUCLEUS & 41 & $1.8 \mathrm{E}-18$ & 7.04E-10 & MsigDB \\
\hline Immune_Kong10_5640genes_ImmPort_ComprehensiveListoflmmune-RelatedGenes & 114 & $3.61 \mathrm{E}-49$ & 7.04E-10 & GeneSigDB \\
\hline Lymphoma_Melendez05_4229genes & 81 & 1.57E-39 & 7.04E-10 & GeneSigDB \\
\hline Breast_Farmer05_3198genes_basal_apocrine_luminal & 66 & $1.08 \mathrm{E}-21$ & $7.04 \mathrm{E}-10$ & GeneSigDB \\
\hline Ovarian_Crijns09_2394Genes_17PathwayPredictor & 57 & 7.94E-30 & 7.04E-10 & GeneSigDB \\
\hline StemCell_Nilsson07_3742genes & 45 & 4.86E-07 & $7.04 \mathrm{E}-10$ & GeneSigDB \\
\hline hsa-miR-19a & 3 & $1.49 \mathrm{E}-05$ & $8.43 \mathrm{E}-09$ & miRecords \\
\hline [hsa-miR-21] & 3 & 0.000116 & $8.43 \mathrm{E}-09$ & miRecords \\
\hline hsa-miR-204 & 3 & 0.000164 & $1.95 \mathrm{E}-08$ & miRecords \\
\hline hsa-miR-21 & 3 & 0.000953 & $2.72 \mathrm{E}-07$ & miRecords \\
\hline hsa-miR-125b & 3 & 0.003089 & $2.72 \mathrm{E}-07$ & miRecords \\
\hline
\end{tabular}

them are cancer-related gene sets. Specifically, "SABATES_COLORECTAL_ADENOMA_DN" and "SABATES_COLORECTAL_ADENOMA_UP" from MSigDB and "Intestine_Vecchi07_1024genes" and "Colon_Kim04_235genes" from GeneSigDB supports the importance of those 100 query genes to colorectal cancer. This case study also shows the complementary nature of MSigDB and
GeneSigDB and thus the benefit of integrating them, which has also been proved by [22]

Case study IV: Building disease-specific gene-set association networks (GSANs) based on gene-set similarities

With the unique top 50 gene sets related to colorectal cancer from disease search and gene search (Table 3 and Table 4), we next investigated the gene-set associations 
Table 4 Top search results of gene-based search from microarray datasets

\begin{tabular}{lllll}
\hline Gene-set Name & Hits & P value & FDR & Data Source \\
\hline SABATES_COLORECTAL_ADENOMA_DN & 58 & $4.57 \mathrm{E}-96$ & $2.76 \mathrm{E}-10$ & MsigDB \\
Breast_Farmer05_3198genes_basal_apocrine_luminal & 35 & $2.91 \mathrm{E}-13$ & $2.76 \mathrm{E}-10$ & GeneSigDB \\
SABATES_COLORECTAL_ADENOMA_UP & 34 & $1.62 \mathrm{E}-57$ & $2.76 \mathrm{E}-10$ & MsigDB \\
Immune_Kong10_5640genes_ImmPort_ComprehensiveListoflmmune-RelatedGenes & 34 & $3.56 \mathrm{E}-08$ & $2.76 \mathrm{E}-10$ & GeneSigDB \\
Leukemia_Pellegrini08_2692genes & 32 & $1.28 \mathrm{E}-15$ & $2.76 \mathrm{E}-10$ & GeneSigDB \\
Intestine_Vecchi07_1024genes & 28 & $3.91 \mathrm{E}-23$ & $2.76 \mathrm{E}-10$ & GeneSigDB \\
Viral_Buonomo11_5307genes & 25 & $6.45 \mathrm{E}-05$ & 0.000109 & GeneSigDB \\
SMID_BREAST_CANCER_LUMINAL_B_DN & 23 & $4.16 \mathrm{E}-19$ & $2.76 \mathrm{E}-10$ & MsigDB \\
Lymphoma_Melendez05_4229genes & 22 & $2.03 \mathrm{E}-06$ & $2.76 \mathrm{E}-10$ & GeneSigDB \\
Colon_Kim04_235genes & 21 & $5.18 \mathrm{E}-30$ & $2.76 \mathrm{E}-10$ & GeneSigDB \\
Breast_Parker09_1918genes_IntrinsicGenes_Compilation & 21 & $1.18 \mathrm{E}-08$ & $2.76 \mathrm{E}-10$ & GeneSigDB \\
\hline
\end{tabular}

between them; 863 associations were found by overlapping the gene symbols between each pair of gene sets, out of which 642 also satisfied Fisher's exact test of a $p$ value and FDR less than 0.05 . A network visualization using Cytoscape [23] is shown in Figure 4. Most of those gene sets are connected to one another, and a few share a large number of genes, suggesting that they form a collaborative unit in colorectal cancer.

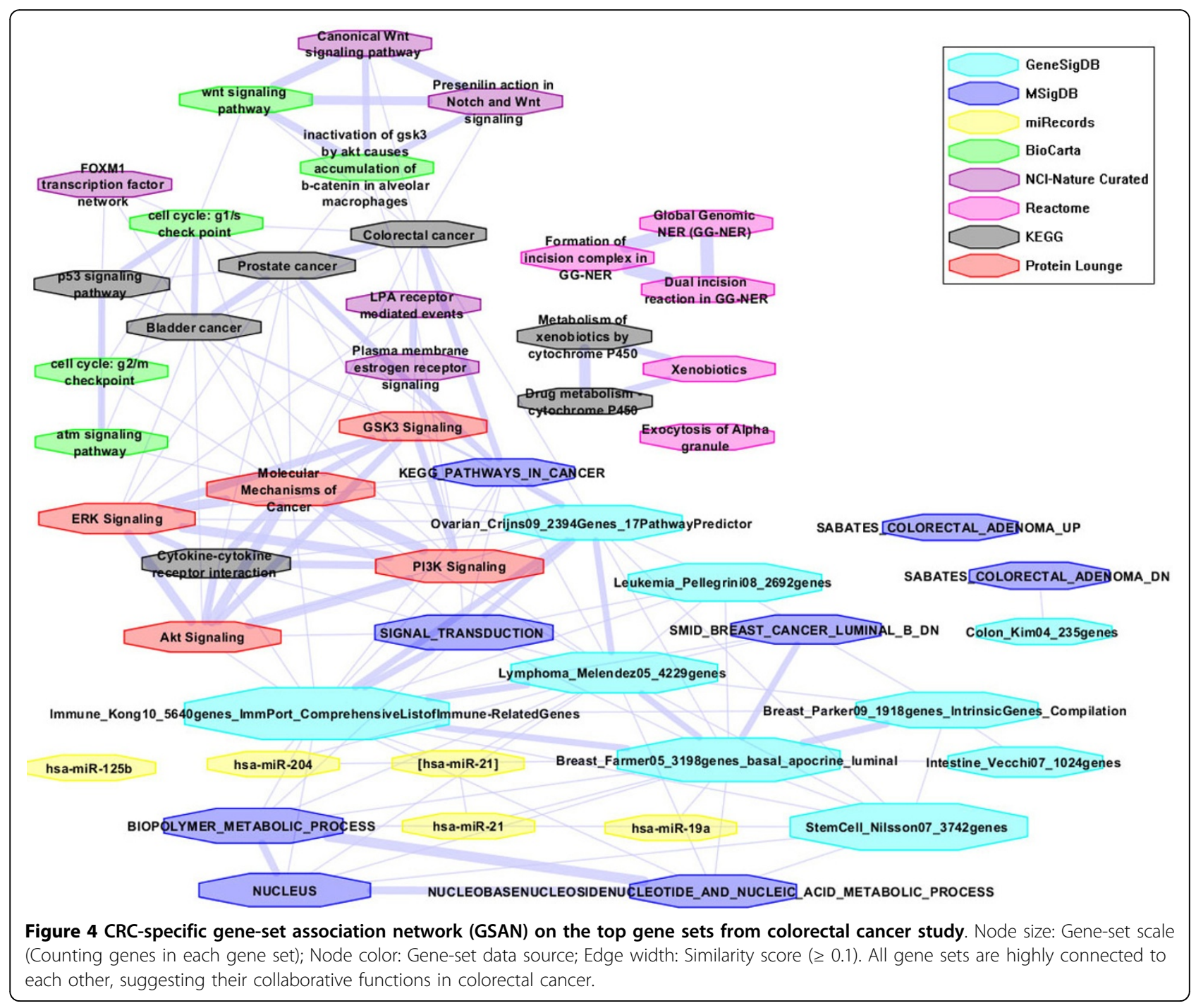




\section{Case study V: Prioritizing disease-associated gene sets by using differential gene-set expressions}

First, the differential gene expression value (ABS_FC) for each gene in a gene set is calculated from the differential analysis based on the microarray data GSE8671. Second, the differential gene-set expression value (NORM_ABS_FC) for each gene set in the CRC-specific GSAN is calculated by using Equation (2). Third, a CRC-specific GSAN with differential gene-set expressions is shown in Figure 5, in which node size represents gene-set scale (Counting genes in each gene set); node color represents differential gene-set expression (NORM_ABS_FC); node line color represents the gene-set data source; and edge width represents the similarity score. By considering differential gene-set expressions for each gene set, we prioritize top-selected gene sets as shown in Table 5. Most of topranked gene sets are closely related to colon tissue, colorectal cancer, or other cancers, which implies that our database can not only support comprehensive diseaseassociated gene-set searching and browsing, but also accurate, disease-specific gene-set prioritizing by using the concept of differential expressions at the gene-set level.

\section{Discussion}

In the near future, we will improve gene-set similarity algorithms by using a global PPI network to calculate their distance. This would provide a more robust measurement for web interface development, and we plan to add a disease browsing function based on disease ontology and a network visualization function to show the gene-set association dynamically. Our final goal is to perform multi-scale network modeling for molecular phenotype discoveries by integrating differential expressions with pathway and network topologies. The current release of PAGED provides a solid foundation for us to develop third-generation pathway analysis tools [1].

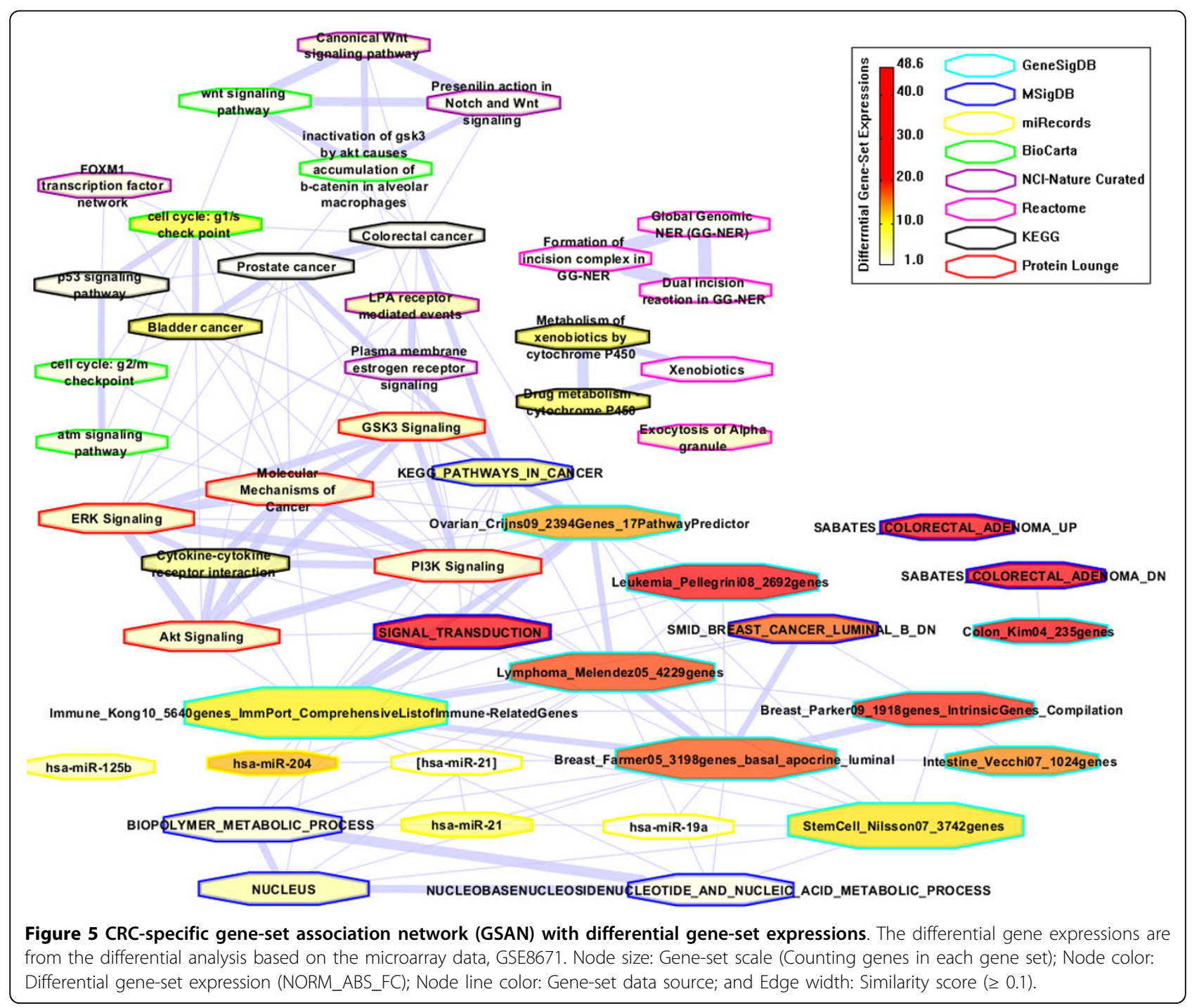


Table 5 Top 20 gene sets ranked by differential gene-set expressions in the CRC-specific gene-set association network (GSAN)

\begin{tabular}{rccc}
\hline Gene-set name & Scale & Data Source & NORM_ABS_FC \\
\hline Colon_Kim04_235genes & 151 & GeneSigDB & 48.58225017 \\
SABATES_COLORECTAL_ADENOMA_DN & 292 & MsigDB & 43.9233159 \\
SIGNAL_TRANSDUCTION & 1598 & MsigDB & 32.5957784 \\
Leukemia_Pellegrini08_2692genes & 2122 & GeneSigDB & 31.65148925 \\
SABATES_COLORECTAL_ADENOMA_UP & 142 & MsigDB & 31.65000681 \\
Breast_Parker09_1918genes_IntrinsicGenes_Compilation & 1734 & GeneSigDB & 20.85621131 \\
Lymphoma_Melendez05_4229genes & 2570 & GeneSigDB & 19.38449282 \\
Breast_Farmer05_3198genes_basal_apocrine_luminal & 3125 & GeneSigDB & 18.93820407 \\
SMID_BREAST_CANCER_LUMINAL_B_DN & 648 & MsigDB & 18.13762096 \\
Intestine_Vecchi07_1024genes & 796 & GeneSigDB & 16.68882931 \\
Ovarian_Crijns09_2394Genes_17PathwayPredictor & 1586 & GeneSigDB & 15.29529767 \\
hsa-miR-204 & 19 & miRecords & 14.37015815 \\
StemCell_Nilsson07_3742genes & 3624 & GeneSigDB & 12.47045771 \\
cell cycle: g1/s check point & 4549 & GeneSigDB & 11.91186233 \\
Bladder cancer & 53 & BioCarta & 9.84279867 \\
Drug metabolism - cytochrome P450 & 89 & KEGG & 7.885181064 \\
Ketabolism of xenobiotics by cytochrome P450 & 94 & KEGG & 7.837851592 \\
hsa-miR-21 & 103 & KEGG & miRecords
\end{tabular}

\section{Conclusions}

We developed PAGED, an online database that provides the most comprehensive public compilation of gene sets. In the current release, PAGED contains a total of 25,242 gene sets, 61,413 genes, 20 organisms, and 1,275,560 records from five major categories: the pathway data from HPD, genome-level disease data from OMIM and GAD, transcriptome-level gene signatures from MSigDB and GeneSigDB, the post-transcriptome microRNA data from miRecords, and proteome-level data from HAPPI. The number of overlapping genes between each data source, gene-set scale distribution, and case study in colorectal cancer shows the synergistic effect of integrating data sources, which greatly facilitate access to gene-setbased prior knowledge. The current PAGED software can help users address a wide range of gene-set-related questions in human disease biology studies.

\section{Additional material}

Additional file 1: Change of similarity score with different $\alpha$ in Equation I. The frequency on the $y$-axis refers to the count of all gene set pairs falling into the category of a particular similarity range on the $x$ axis. Different $\alpha$ in Equation I have been used to calculate the similarity score. When $\alpha$ approaches 0 , the distribution skewed to right with many false positive high similarity scores; when $\alpha$ approach 1, the distribution is too left-skewed failing to differentiate those low similarity scores apart.

\section{Acknowledgements}

We would like to thank MedeoLinx, LLC for their financial support. We also thank Indiana University Collaborative Research Grants (IUCRG), Indiana University-Purdue University Indianapolis (IUPUI) Solution Center, Multidisciplinary Undergraduate Research Institute (MURI) and Indiana Center for Systems Biology and Personalized Medicine (CSBPM) for their support. This article has been published as part of BMC Bioinformatics Volume 13 Supplement 15, 2012: Proceedings of the Ninth Annual MCBIOS Conference. Dealing with the Omics Data Deluge. The full contents of the supplement are available online at http://www.biomedcentral.com/bmcbioinformatics/ supplements/13/S15

\section{Author details}

${ }^{1}$ School of Informatics, Indiana University, Indianapolis, IN 46202, USA. ${ }^{2}$ Indiana Center for Systems Biology and Personalized Medicine, Indiana University, Indianapolis, IN 46202, USA. ${ }^{3}$ MedeoLinx, LLC, Indianapolis, IN 46280, USA. ${ }^{4}$ Capital Normal University, Beijing, 100048, China.

\section{Authors' contributions}

JYC conceived of this work, guided the research team by providing ideas and feedback along the way, and revised the manuscript. $\mathrm{HH}$ integrated disease-gene association data, developed the website, designed the case studies and wrote the manuscript. XW participated in the idea initiation, framework development, data quality control, case studies, and manuscript writing. MS integrated various pathways, microRNA, and gene signature data. SNM reviewed the evolvement on pathway analysis and gene-set enrichment analysis. RP helped with the database management and maintenance. KFM tested the website, provided valuable suggestions for substantial improvements, and revised the manuscript. PW assisted with website maintenance. All authors read and approved the final manuscript.

\section{Competing interests}

The authors declare that they have no competing interests.

Published: 11 September 2012 


\section{References}

1. Khatri P, Sirota $M$, Butte AJ: Ten years of pathway analysis: current approaches and outstanding challenges. PLOS computational biology 2012, 8(2):e1002375

2. Holmans P: Statistical methods for pathway analysis of genome-wide data for association with complex genetic traits. Advances in genetics 2010, 72:141.

3. Ramanan VK, Shen L, Moore JH, Saykin AJ: Pathways analysis of genomic data: concepts, methods, and prospoects for future development. Trends in Genetics 2012

4. Slonim DK: From patterns to pathways: gene expression data analysis comes of age. Nature genetics 2002, 32(supp):502-508.

5. Abatangelo L, Maglietta R, Distaso A, D'Addabbo A, Creanza TM, Mukherjee S, Ancona N: Comparative study of gene set enrichment methods. Bmc Bioinformatics 2009, 10(1):275.

6. Subramanian A, Tamayo P, Mootha VK, Mukherjee S, Ebert BL, Gillette MA, Paulovich A, Pomeroy SL, Golub TR, Lander ES: Gene set enrichment analysis: a knowledge-based approach for interpreting genome-wide expression profiles. Proceedings of the National Academy of Sciences of the United States of America 2005, 102(43):15545.

7. Culhane AC, Schroder MS, Sultana R, Picard SC, Martinelli EN, Kelly C, HaibeKains B, Kapushesky M, St Pierre AA, Flahive W, et al: GeneSigDB: a manually curated database and resource for analysis of gene expression signatures. Nucleic acids research 2012, 40:D1060-1066.

8. Liberzon A, Subramanian A, Pinchback R, Thorvaldsdottir H, Tamayo P, Mesirov JP: Molecular signatures database (MSigDB) 3.0. Bioinformatics 2011, 27(12):1739-1740.

9. Eleftherohorinou $H$, Wright $V$, Hoggart $C$, Hartikainen $A L$, Jarvelin MR, Balding D, Coin L, Levin M: Pathway analysis of GWAS provides new insights into genetic susceptibility to 3 inflammatory diseases. PLoS One 2009, 4(11):e8068.

10. Wang K, Li M, Bucan M: Pathway-based approaches for analysis of genomewide association studies. Am J Hum Genet 2007, 81(6):1278-1283.

11. Zhong H, Yang X, Kaplan LM, Molony C, Schadt EE: Integrating pathway analysis and genetics of gene expression for genome-wide association studies. Am J Hum Genet 2010, 86(4):581-591.

12. Chowbina SR, Wu X, Zhang F, Li PM, Pandey R, Kasamsetty HN, Chen JY: HPD: an online integrated human pathway database enabling systems biology studies. BMC bioinformatics 2009, 10(Suppl 11):S5.

13. Chowbina S, Deng Y, Ai J, Wu X, Guan X, Wilbanks MS, Escalon BL, Meyer SA, Perkins EJ, Chen JY: A new approach to construct pathway connected networks and its application in dose responsive gene expression profiles of rat liver regulated by 2, 4DNT. BMC genomics 2010, 11(Suppl 3):S4.

14. Hamosh A, Scott AF, Amberger JS, Bocchini CA, McKusick VA: Online Mendelian Inheritance in Man (OMIM), a knowledgebase of human genes and genetic disorders. Nucleic acids research 2005, 33:D514-517.

15. Becker KG, Barnes KC, Bright TJ, Wang SA: The genetic association database. Nature genetics 2004, 36(5):431-432.

16. Xiao F, Zuo Z, Cai G, Kang S, Gao X, Li T: miRecords: an integrated resource for microRNA-target interactions. Nucleic acids research 2009, 37: D105-110.

17. Chen JY, Mamidipalli S, Huan T: HAPPI: an online database of comprehensive human annotated and predicted protein interactions. BMC genomics 2009, 10(Suppl 1):S16.

18. Pang-Ning $T$, Steinbach $M$, Kumar $V$ : Introduction to data mining. Boston: Person Addison Wesley EducatioPress; 2005.

19. Jothi R, Przytycka TM, Aravind L: Discovering functional linkages and uncharacterized cellular pathways using phylogenetic profile comparisons: a comprehensive assessment. BMC bioinformatics 2007, 8(1):173-117.

20. Edgar R, Domrachev M, Lash AE: Gene Expression Omnibus: NCBI gene expression and hybridization array data repository. Nucleic acids research 2002, 30(1):207-210.

21. Segditsas S, Tomlinson I: Colorectal cancer and genetic alterations in the Wnt pathway. Oncogene 2006, 25(57):7531-7537.

22. Sonachalam Madhankumar, Shen Jeffrey, Huang Hui, Wu Xiaogang: Systems biology approach to identify gene network signatures for colorectal cancer. Frontiers in Genetics 2012, 3:80.
23. Smoot ME, Ono K, Ruscheinski J, Wang PL, Ideker T: Cytoscape 2.8: new features for data integration and network visualization. Bioinformatics 2011, 27(3):431-432.

doi:10.1186/1471-2105-13-S15-S2

Cite this article as: Huang et al:: PAGED: a pathway and gene-set enrichment database to enable molecular phenotype discoveries. BMC Bioinformatics 2012 13(Suppl 15):S2.

\section{Submit your next manuscript to BioMed Central and take full advantage of:}

- Convenient online submission

- Thorough peer review

- No space constraints or color figure charges

- Immediate publication on acceptance

- Inclusion in PubMed, CAS, Scopus and Google Scholar

- Research which is freely available for redistribution

Submit your manuscript at www.biomedcentral.com/submit
C Biomed Central 